Journal of Epidemiology and Community Health, 1980, 34, 316-317

To the Editor

\section{Mortality and two indicators of morbidity}

SIR-The article by Brennan and Clare $^{1}$ seeks to demonstrate the relationship between two indicators of self-reported morbidity derived from the 1971 census and age-band specific mortality data for all causes of death at a level of aggregation below county level. They argue from the strength and nature of this relationship that it is reasonable to use mortality data in the RAWP formula applied to area or health district level.

The conclusions of the paper are challenged on three grounds: (1) The data available is unsuited to the hypothesis being discussed; (2) The interpretation of the results is of doubtful statistical validity; and (3) The logic of the argument is open to question.

The authors acknowledge that the morbidity data available are restricted to persons expected to be economically active and relate only to self-reported conditions which prevent economic activity. The population is thus composed mainly of males of working age, a group which is a relatively low user of health service resources. The authors concede that the morbidity data for the group ' 65 years and over' are unreliable in that these represent only a small part of the total morbidity spectrum, but proceed to make an extrapolation of doubtful validity from the morbidity experience of a younger age group. They further acknowledge that the relationship of the indicators to the total spectrum of morbidity for all age groups did not appear to be constant at each geographical data point. The absence of data for children aged 0-15 years precludes the use of standardised mortality ratios; the width of age-banding required to match the two sets of data for age-specific rates is, however, such that standardisation for age is minimal. In view of these major reservations concerning the data, it is surprising that the authors felt justified in proceeding.

The interpretation of the results is contentious and in our view is of doubtful statistical validity.

We note with interest that the scattergram supplied for the age-group 45-64 years is 'of the most highly correlated data'. The overall impression is misleading in that it does not reveal the strength of the relationship for the lower values of the morbidity range in which most of the data points lie. Neither can it indicate the considerable variation which may occur among narrow age bands with the age-group as a whole.

The interpretation of the numerical values of the correlation coefficients, which are in effect only summary statistics, is notoriously difficult. The estimation of the likelihood of such values having occurred by chance alone is also suspect, being dependent upon the assumption of bivariate normality. No evidence for such an assumption has been introduced. In our opinion, there is a high probability that the distribution of both morbidity and mortality data is skewed by the effect of socioeconomic factors at the various geographical data points. The findings would have been more convincing if supported by a similarly high degree of correlation obtained by the use of a non-parametric test free from assumptions regarding distribution.

As the authors state, the use of the SMR in the RAWP formula has considerable implications for resource allocation. Its use, however, is not as implied, as an overall multiplier using the all-condition SMR, but on a grouped-condition specific basis. At a lower level of geographical aggregation, the problems we have noted before regarding the very small numbers of deaths in certain groups become readily apparent.

The authors have used throughout their paper and calculations age-specific mortality rates in broad age bands. The RAWP formula uses SMRs which are disease-group-specific. At no point can we find an explanation in the paper for the assumption that the effect of these will be the same.

In view of the above problems, the relationship claimed for morbidity and mortality must be regarded as not proven and the evidence insufficient to support the use of mortality data for resource allocation below regional levels. We welcome however, the final conclusion that the RAWP formula should be tempered by planning aspirations. $\subseteq$

(Dr.) H. P. FERRER Area Medical Office G. STEVENS

Lecturer in Information Systems/O.R Warwick University

(Dr.) A. MOORE Area Specialist in Community Medicine (Planning and Information)

Hereford and Worcester Area Health Authority Love's Grove, Castle Street, Worcester WR1 3BZ.

\section{Reference}

${ }^{1}$ Brennan ME, Clare $\mathrm{PH}$. The relationship between mortality and two indicators of morbidity. $J$ Epidemiol Community Health 1980; 34: 134-8.

\section{Brennan and Clare reply:}

Available data and other research. Unlike the reviewers of our article we did not expect that one piece of research would answer all or even the majority of questions raised by the use of SMRs in the RAWP formula. ${ }^{1}$ We believe that our results provide a useful addition to the accumulating evidence on mortality and morbidity relationships. It is true that our data allowed conclusions to be drawn only for the working population in the age range 16-64 years. However, other research has indicated similar conclusions for children.

It is fairly well documented that in certain social groups where common causes of death in children 
have a high incidence, that is, respiratory disease, congenital anomalies, and perinatal causes, the morbidity from these disorders is also increased. For example, in respiratory disorders the factors implicated are external pollution, social class, overcrowding, and poverty. ${ }^{2}$ Wynn and $\mathrm{Wynn}^{3}$ in an informative review show that social groups with high mortality rates from perinatal causes and congenital malformations also have increased morbidity.

In adults of working age external factors more commonly found in certain localities than others affect both mortality and morbidity; for example, lower socioeconomic groups have overall both higher mortality and morbidity. ${ }^{4}$ In common specific diseases an increased morbidity was found to be a very good predictor of subsequent mortality. ${ }^{5}$ In the light of this evidence and that presented in our paper, it seems reasonable to assume that there is a positive relationship between the morbidity experience and mortality experience of a group in a defined geographical area. It is of course not possible to state the exact relationship which would be expected in any given geographical area and it would be unreasonable to expect any statistical model to do this. We would agree that our morbidity indicators measure only disability severe enough to justify absence from work. However, we would regard this restriction as useful in limiting the cases involved to those which have serious social consequences.

About $50 \%$ of inpatient hospital resources are consumed by the elderly, so this is clearly a population group for which measures of need should be developed and incorporated in resource allocation. Our work, we agree, says little about this age group.

We recommended the use of age-specific mortality as readily available indicators and not measures of morbidity.

Non-parametric tests. The reviewers state that our results would be more convincing if a non-parametric test, free from assumptions about the underlying distributions, had been used. As a matter of fact, the first test we used in our research was non-parametric; we reported on the results in earlier drafts of our paper, but they were later omitted to reduce the length of the paper. We used Kendall's rank correlation test, with correction for tied ranks. The standard error of Kendall's coefficient has been derived and it is known that for numbers of observations greater than 30 (a condition met by all our data sets) it is distributed normally.

Results of non-parametric test:-Kendall's rank correlation. The rank correlation coefficients were, in general, as statistically significant as those obtained from linear regression. Complete agreement occurred for the 45-64 age group. For the other two age groups, Kendall's test showed sometimes higher and sometimes lower levels of significance, but in no case was the level of significance below 95\%. Nearly all the differences occurred with the permanently sick morbidity measure. As the reviewers suggest, the scattergram shown in the paper could be misleading, because a few points at the high morbidity end of the scale could be exerting undue influence. Rank correlation removes distortions of this type, and yet in our case it confirmed the inferences given in the paper. (Detailed tables are available on request).

Finally, there is a large body of evidence that mortality and morbidity rates are higher in the northern regions than in the south, while National Health Service resources allocated to these regions are still substantially below the national average. While the scientific basis for SMRs is still incomplete they do have the advantage of recognising the relative deprivation which exists. Some of the districts in our own region are 15-20\% below RAWP target, so whatever one's views on the details of the application of the RAWP formula these calculations clearly imply severe deprivation. To those regions and districts who are well off in RAWP terms, the SMR represents a threat. However, this is only true if the growth rate is minimal.

It is important to remember that the proportion of the GNP spent in this country on health is substantially below that of other similarly developed countries. ${ }^{6}$ Any marked relief of the deprivation which occurs in some districts can occur only if an increase of $3 \%$ per annum at least is envisaged for approximately 10 years. We would suggest that rather than redistributing resources within a fixed budget an overall increase in expenditure on the NHS is highly desirable.

M. E. BRENNAN

P. H. CLARE

West Midlands Regional Health Authority,

Arthur Thomson House,

146 Hagley Road,

Birmingham B16 9PA.

\section{References}

${ }^{1}$ Kuhn T. The Structure of Scientific Revolutions. London: University of Chicago Press, 1962.

${ }^{2}$ Leeder SR, Holland WW. The influence of the environment on disease and growth in children. In: Bennett AE, ed. Recent Advances in Community Medicine. London: Churchill Livingstone, 1978.

${ }^{3}$ Wynn M, Wynn A.Prevention of Handicap and the Health of Women. London: Routledge and Kegan Paul, 1979.

${ }^{4}$ Morris JN. Social inequalities undiminished. Lancet 1979; i: 87.

${ }^{5}$ Todd G, Hunt BM, Lambert PM. Four cardiorespiratory symptoms as predictors of mortality. J Epidemiol Community Health 1978; 32: 267-74.

${ }^{6}$ Office of Health Economics. Briefing Paper No. 10. Perinatal Mortality in Britain:-a question of class. London: Office of Health Economics, 1979. 